\title{
Carbon Dioxide-Induced Emotion and Respiratory Symptoms in Healthy Volunteers
}

\author{
Alessandro Colasanti', Ewa Salamon', Koen Schruers', Rob van Diest', Marlies van Duinen', Eric J Griez ${ }^{*, I}$ \\ 'Department of Psychiatry and Neuropsychology, University of Maastricht, Maastricht, The Netherlands
}

\begin{abstract}
A number of evidences have established that panic and respiration are closely related. Clinical studies indicated that respiratory sensations constitute a discrete cluster of panic symptoms and play a major role in the pathophysiology of panic. The aim of the present study was to explore the phenomenology of an experimental model of panic in healthy volunteers based on the hypothesis that: (I) we can isolate discrete clusters of panic symptoms, (2) respiratory symptoms represent a distinct cluster of panic symptoms, and (3) respiratory symptoms are the best predictor of the subjective feeling of panic, as defined in the DSM IV criteria.Sixty-four healthy volunteers received a double inhalation of four mixtures containing $0,9,17.5$ and $35 \% \mathrm{CO}_{2}$, respectively, in a double-blind, cross-over, random design. An electronic visual analog scale and the Panic Symptom List (PSL) were used to assess subjective 'fear/discomfort' and panic symptoms, respectively. Statistical analyses consisted of Spearman's correlations, a principal component factor analysis of the 13 PSL symptoms, and linear regressions analyses. The factor analysis extracted three clusters of panic symptoms: respiratory, cognitive, and neurovegetative $\left(r^{2}=0.65\right)$. Respiratory symptoms were highly related to subjective feeling of fear/discomfort specifically in the $\mathrm{CO}_{2^{-}}$ enriched condition. Moreover, the respiratory component was the most important predictor of the subjective feeling of 'fear/discomfort' $(\beta=0.54)$.The discrete clusters of symptoms observed in this study were similar to those elicited in panic attacks naturally occurring in patients affected by panic disorder. Consistent with the idea that respiration plays a crucial role in the pathophysiology of panic, we found that respiratory symptoms were the best predictors the subjective state defined in the DSM IV criteria for panic.

Neuropsychopharmacology (2008) 33, 3103-31 10; doi: 10.1038/npp.2008.31; published online 19 March 2008
\end{abstract}

Keywords: carbon dioxide; respiration; panic; emotion; anxiety

\section{INTRODUCTION}

Panic attacks (PAs) are defined in the current nosology as discrete periods of intense fear or discomfort accompanied by diverse physical and cognitive symptoms (APA, 2000). The symptomatology includes cardio-respiratory symptoms (dyspnea or breathlessness, palpitations, and chest pain); neurovegetative symptoms (nausea and sweating), and other nonspecific symptoms (trembling, paresthesias, dizziness, and chills). Frequent cognitive symptoms include feelings of derealization or depersonalization, fear of losing control or going crazy, and fear of dying.

Research from the past two decades has focused considerable attention on elucidating the phenomenology of panic, resulting in the recognition that PAs are characterized by inter-individual heterogeneity. Researchers have identified distinct subtypes of panic disorder (PD) on the basis of a predominant symptom constellation (Bass

\footnotetext{
*Correspondence: Dr E Griez, Department of Psychiatry and Neuropsychology, Maastricht University, PO Box 616, Maastricht 6200 AB, The Netherlands, Tel: + 31433685 332, Fax: + 31433685 317, E-mail: eric.griez@pn.unimaas.nl

Received 6 November 2007; revised 29 January 2008; accepted 8 February 2008
}

et al, 1987; Aronson and Logue, 1988; Ley, 1992). Such an approach may contribute to a better understanding of the etiology of panic since dysregulation in different psychobiological mechanisms may underlie phenotypically similar attacks. Gorman and co-workers in 1999 posed a neuroanatomical hypothesis of PD based on the idea that the physiological and behavioral consequences of a panic attack are mediated by a 'central fear network' in the brain that is centered in the amygdala and involves its interaction with the hippocampus, medial prefrontal cortex, and hypothalamic and brainstem sites (Gorman et al, 2000).

Even though clustering of panic symptoms has not yielded always consistent results, there is general agreement about the existence of a prominent respiratory subtype of panic that is characterized by the prevalence of respiratory symptoms. Briggs et al (1993) analyzed a sample of 1168 PD patients and divided them into two clusters. The first cluster, labeled 'respiratory', was characterized by the presence of shortness of breath, choking, fear of dying, chest pain, and tingling; the second 'nonrespiratory' cluster was characterized by the absence of respiratory symptoms and the presence of the other symptoms listed in DSM-IV criteria for panic attack. Consistently with Briggs, Argyle and Roth (1989) and De Beurs et al (1994) found a similar 'respiratory' cluster in their factor analysis. Cox et al (1994) 
extracted three factors: a dizziness-related symptom cluster, a cardio-respiratory distress symptom cluster, and a catastrophic cognition symptom cluster. The cardiorespiratory cluster was primarily characterized by palpitations but also included dyspnea, choking, and fear of dying. Recently, Meuret et al (2006) analyzed 343 PD patients and extracted three 'dimensions' interpreted as cardio-respiratory, mixed somatic-autonomic, and cognitive. The cardiorespiratory cluster included shortness of breath, chest pain, fear of dying, palpitations, tingling, and choking. Shioiri et al (1996), in a Japanese study of 207 PD patients, found slightly different results. Their cluster analysis revealed three panic symptom clusters. The first was comprised exclusively of physiological symptoms, among which respiratory symptoms were prominent. The second included mixed panic symptoms, including agoraphobia and anticipatory anxiety, and the third was comprised of fearful cognitive symptoms plus paresthesias and chest pain.

The existence of a distinct 'prominent respiratory' subtype of panic seems to be supported by clinical evidence. Briggs et al (1993) demonstrated that patients with the respiratory subtype of panic suffer more spontaneous PAs than patients with the residual subtype and that they respond better to imipramine than to alprazolam. The respiratory subtype has been shown to be associated with increased familial risk of PD (Horwath et al, 1997; Nardi et al, 2003, 2006a, b), to be more common among heavy smokers, to have a longer duration and major severity of illness (Biber and Alkin, 1999), and to have a lower resting end-tidal $\mathrm{pCO}_{2}$ (Moynihan and Gevirtz, 2001) and a higher sensitivity to $\mathrm{CO}_{2}$ inhalation (Biber and Alkin, 1999; Valenca et al, 2002; Abrams et al, 2006; Nardi et al, 2006a, b). Other clinical features displayed by PD patients with the respiratory subtype include a later onset of the disease and a faster response to nortriptyline (Nardi et al, 2003, 2006a, b), more past traumatic suffocation experiences and respiratory diseases (Bouwer and Stein, 1997; Verburg et al, 1995), higher levels of anxiety sensitivity, and more panic-agoraphobic spectrum symptoms (Onur et al, 2006).

A strong relation between panic and respiration would be consistent with the high co-morbidity of respiratory disorders (asthma, COPD) and PD observed in large, epidemiological studies (Goodwin and Pine, 2002). Taken together, the data emerging from clinical, experimental, and epidemiological studies on panic and respiration suggest that the neurobiological mechanism underlying PD may involve respiratory control (Klein, 1993; Preter and Klein, 2008; Battaglia and Ogliari, 2005).

Briggs, Meuret, Biber, Nardi and coworkers have suggested that: (1) panic symptoms can be grouped into clusters, (2) respiratory symptoms constitute a distinct and well-defined cluster, and (3) respiratory symptoms play a major role in its pathophysiology.

In the present study, we sought evidence to support these suggestions by analyzing an experimental model of panic. We recently showed that increasing concentrations of $\mathrm{CO}_{2}$ dose dependently induce a negative affect, which eventually culminates in genuine panic, also in healthy individuals (Griez et al, 2007). Accordingly, high doses of $\mathrm{CO}_{2}$ may be considered as a model of panic in healthy subjects, just as moderate doses are in PD patients. On these premises, we performed a statistical analysis of the symptom pattern observed in our 2007 dose response experiment. In line with the above mentioned authors, we hypothesized that: (1) there are discrete clusters of panic symptoms, (2) respiratory symptoms can be extracted as an independent component, and (3) respiratory symptoms are, indeed, the best predictor of the subjective disturbance defined in the DSM IV criteria for panic.

\section{MATERIALS AND METHODS}

\section{Subjects}

Students and staff members were recruited by word of mouth from within Vijverdal Psychiatric Hospital in Maastricht and from the University of Maastricht. They received course credit for their participation in the studies. All potential participants provided a complete medical history and underwent a physical examination. Inclusion criteria were age 18-65 years and a good present and past physical and mental condition, based on the results of a structured psychiatric interview (Mini International Neuropsychiatric Interview) performed by a psychologist not directly involved in the study. Exclusion criteria included a history of pulmonary or cardiovascular disease, the presence of hypertension (diastolic > $100 \mathrm{~mm} \mathrm{Hg}$; systolic > $170 \mathrm{~mm} \mathrm{Hg}$ ), cerebral aneurysm, pregnancy, epilepsy, excessive smoking ( $>15$ cigarettes/day), use of adrenergic receptor blockers, and use of psychotropic medication. Individuals with a history of affective or anxiety disorders in a first-degree relative were excluded. Individuals with a present or past history of substance-related disorders, including caffeine-related disorder, were excluded. Participants were also excluded if they reported common specific fears or if they had a history of PAs. The ethics committee of the University of Maastricht approved the study. A complete description of the study was given both orally and in written form to the subjects. Those who met the inclusion criteria and who provided written informed consent were included in the study.

\section{Procedure}

The study was conducted according to a double-blind, randomized, cross-over design. All subjects received all of the following challenges: air: $0 \% \mathrm{CO}_{2}, 20 \% 0_{2}, 80 \% \mathrm{~N}_{2}$; low: 9\% $\mathrm{CO}_{2}, 20 \% 0_{2}, 71 \% \mathrm{~N}_{2}$; medium: $17.5 \% \mathrm{CO}_{2}, 20 \% 0_{2}$, $62.5 \% \mathrm{~N}_{2}$; high: $35 \% \mathrm{CO}_{2}, 20 \% 0_{2}, 55 \% \mathrm{~N}_{2}$ in randomized order. Each subject underwent the four different challenges on four different days within the same week, at the same hour, to eliminate circadian effects in the challenge response. Every challenge was performed in the morning, between 0900 and $1200 \mathrm{~h}$. The participants were asked to refrain from drinking beverages containing xanthine for at least $8 \mathrm{~h}$ and from eating for at least $2 \mathrm{~h}$ before the test.

The procedure was standardized for all subjects and for all sessions and was performed according to the protocol for $35 \% \mathrm{CO}_{2}$ inhalation used at the Maastricht Academic Anxiety Center (Griez et al, 1987). The different gas mixtures were all delivered through the same nasal-oral exercise self-administration face mask using a double vital capacity inhalation technique. Before the challenge was started, the inspired vital capacity of every subject was 
evaluated using an analog respirometer (Wright respirometer Mark 20) connected to the self-administration mask. The same respirometer measured the gas volume delivered at each inhalation. Baseline inspired vital capacity with a double breath of normal air was measured on each occasion, and a test breath was considered adequate if it was more than $80 \%$ of the baseline vital capacity. The subjects were then given the self-administration mask and told to hold it in their hands and to exhale as deeply as they could. A double-breath inhalation of a mixture containing one of the four different concentrations of $\mathrm{CO}_{2}$ was then delivered to the subjects. They were asked to take a maximal inspiration through the self-administration mouthpiece and to make a complete expiration outside the mouthpiece, immediately followed by a second maximal inspiration through the mouthpiece following the same procedure. At the end of the second inhalation, the subjects were asked to hold their breath for $4 \mathrm{~s}$ to enhance the alveolar gas exchange.

\section{Measures}

Assessments were chosen with strict reference to the definition of PA in the current psychiatric nosology, and specifically the DSM IV TR criteria for PA. These define a $\mathrm{PA}$ as a discrete period of intense fear or discomfort followed by the abrupt development of 13 well-defined symptoms. An electronic visual analog scale for affect (eVAAS) was developed to record the subjective feeling of fear or discomfort. It consists of a Compaq laptop touch screen personal computer interfaced with original eVAAS software, in which the analog scale is shown on the electronic display and the subject interacts directly via an electronic pen with the touch screen. The top of the display is labeled 'fear or discomfort'. The participants were instructed to indicate the amount of the subjective disturbance, in case of feeling either fear or discomfort. The visual analog scale is represented by a $200-\mathrm{mm}$ bar on the display that ranges from 0 (no fear/discomfort at all) to 100 (the worst imaginable fear/discomfort). This instrument has been validated for use during $35 \% \mathrm{CO}_{2}$ challenges (Van Duinen et al, 2008).

Before each challenge began, the participants rated their baseline level of fear/discomfort.

Panic symptoms were evaluated using the Panic Symptom List (PSL-IV; Schruers et al, 2000). This consists of a questionnaire listing 13 items, each representing one of the DSM-IV TR symptoms (ie, palpitations; sweating; trembling; sensations of shortness of breath or smothering; feeling of choking; chest discomfort; nausea or abdominal distress; feeling dizzy, lightheaded, or faint; derealization or depersonalization; fear of losing control; fear of dying; paresthesias; chills or hot flushes). The participants were asked to rate the intensity of each symptom from 0 (absent) to 4 (very intense). The total scores thus ranged from 0 to 52 .

Baseline assessment of eVAAS and PSL-IV symptoms were obtained one minute before each double inhalation (baseline score). One minute after the end of each double inhalation, the subjects filled in both the eVAAS and the PSL-IV questionnaire. At that time, they were asked to indicate the worst moment they experienced after inhaling the gas mixture (post-inhalation score).

The post-inhalation change was calculated by subtracting the baseline score from the post-inhalation score.

\section{Statistical Analysis}

The statistical analyses were performed using SPSS 13.0. First, an ANOVA for repeated measures was conducted on the eVAAS score, total PSL score, and each of PSL symptom scores. The subsequent analysis of the relationship between eVAAS score and PSL symptoms consisted of four steps:

(1) Spearman's non-parametric correlation analysis between eVAAS score and each of the 13 PSL-IV symptoms;

(2) First linear regression analysis to determine the relative weight of each PSL-IV symptom in the determination of the eVAAS score. The dependent variable was the eVAAS score and the predicting variables were the PSL-IV symptoms;

(3) Principal component factor analysis with varimax rotation to obtain variables with very high factor weights in some factors and very low factor weights in others. To insert a variable into a factor, a factor weight of at least 0.5 was selected as the threshold value. Factor scores were then computed as variables, representing a measure of each subject's contribution to each factor.

(4) Second linear regression analysis to determine the relative weight of each principal component in the determination of the eVAAS score. The dependent variable was the eVAAS score and the predicting variables were the principal factor scores.

The correlation analysis and first linear regression analysis were made separately for each condition (air, low, medium, high). The principal component factor analysis was applied to the 13 PSL-IV symptoms only in the high condition $\left(35 \% \mathrm{CO}_{2}\right)$. The second linear regression analysis was performed only in the high condition.

\section{RESULTS}

Sixty-four volunteers were included in the study: 33 males and 31 females aged 35. 8 (15.9 SD) and 31.1 (14.4 SD) years, respectively.

PSL-IV scores for each symptom are presented in Table 1. Dizziness, sensation of shortness of breath, feeling of choking, and palpitations had the highest mean scores in all three $\mathrm{CO}_{2}$ conditions (high, medium, and low). For both the eVAAS score and the PSL-IV total score, a statistically significant dose-response relationship was observed (these results are extensively presented elsewhere) (Griez et al, 2007). Each PSL symptom score was significantly dosedependent $(p<0.05)$.

\section{Correlations}

Correlation coefficients are shown in Table 2. A high Spearman's rho coefficient was found between the symptoms 'feeling of choking' and 'sensation of shortness of 
breath' in all conditions except air. The symptoms 'derealization or depersonalization', 'fear of losing control', and 'fear of dying' were highly related to each other in all of the conditions.

Table I Panic Symptom List Scores

\begin{tabular}{|c|c|c|c|c|c|c|c|c|}
\hline \multirow{2}{*}{ DOSE } & \multicolumn{2}{|c|}{ Air } & \multicolumn{2}{|c|}{ Low } & \multicolumn{2}{|c|}{ Medium } & \multicolumn{2}{|c|}{ High } \\
\hline & Mean & SD & Mean & SD & Mean & SD & Mean & SD \\
\hline Palpitations & 0.47 & 0.73 & 0.50 & 0.70 & 0.69 & 0.76 & 1.31 & 1.06 \\
\hline Sweating & 0.00 & 0.44 & 0.10 & 0.56 & 0.25 & 0.54 & 0.63 & 0.81 \\
\hline Trembling & 0.14 & 0.43 & 0.16 & 0.52 & 0.30 & 0.69 & 0.81 & 0.97 \\
\hline Shortness of breath & 0.30 & 0.55 & 0.37 & 0.73 & 0.72 & 0.82 & 1.47 & 1.16 \\
\hline Feeling of choking & 0.20 & 0.44 & 0.40 & 0.73 & 0.67 & 0.79 & 1.56 & 1.13 \\
\hline Chest pain & 0.02 & 0.22 & 0.05 & 0.28 & 0.07 & 0.31 & 0.39 & 0.71 \\
\hline Nausea & 0.09 & 0.50 & 0.05 & 0.28 & 0.08 & 0.33 & 0.29 & 0.82 \\
\hline Dizziness & 0.72 & 0.92 & 0.63 & 0.71 & 1.03 & 0.84 & 1.74 & 1.13 \\
\hline Derealization & 0.17 & 0.38 & 0.18 & 0.43 & 0.21 & 0.58 & 0.66 & 0.90 \\
\hline Fear of losing control & 0.05 & 0.21 & 0.02 & 0.22 & 0.16 & 0.49 & 0.29 & 0.66 \\
\hline Fear of dying & 0.00 & 0.00 & 0.02 & 0.13 & 0.02 & 0.13 & 0.10 & 0.39 \\
\hline Paresthesias & 0.14 & 0.39 & 0.11 & 0.32 & 0.20 & 0.48 & 0.65 & 1.03 \\
\hline Chills & 0.06 & 0.24 & 0.02 & 0.34 & 0.08 & 0.33 & 0.19 & 0.54 \\
\hline PSLtot & 2.47 & 3.07 & 2.60 & 3.51 & 4.48 & 4.01 & 10.08 & 7.07 \\
\hline E-VAAS & 16.06 & 17.98 & 17.66 & 18.56 & 26.16 & 24.19 & 44.94 & 26.62 \\
\hline
\end{tabular}

DSM panic symptoms intensity (mean $\pm \mathrm{SD}$ ) in four different $\mathrm{CO}_{2}$ conditions.
Correlation coefficients between eVAAS score and PSL-IV scores across the four different doses are presented in Figure 1. Dizziness and palpitations were strongly related to the eVAAS score in all of the $\mathrm{CO}_{2}$ conditions, including the air condition. Sensation of shortness of breath and feeling of choking were strongly related to the eVAAS score in all of the $\mathrm{CO}_{2}$ conditions but not in the air condition.

\section{Regression Analysis (1)}

A regression analysis was performed to show the weight of each symptom in predicting the eVAAS score. Figure 2 shows the relative weight ( $\beta$ score) of each PSL-IV symptom separately for the four conditions. In the high condition, 'feeling of choking' had the highest $\beta$ score. 'Sensation of shortness of breath' appeared to be a poor predictor. Looking at the other conditions, it is evident that the weight of 'feeling of choking' in predicting the eVAAS score increased with the dose, while the weight of 'shortness of breath' decreased with the dose. In the air condition, both respiratory symptoms had a low predicting value $(<0)$ compared to 'palpitations', 'chest pain', and 'dizziness', which had $\beta$ scores higher than 0.3 .

\section{Factor Analysis}

The factor analysis extracted and rotated three components - respiratory, cognitive, and neurovegetative - which accounted for over $65 \%$ of the variance (Table 3). The respiratory component was mainly characterized by 'sensation of shortness of breath' and 'feeling of choking'. It also

Table 2 Correlations between Panic Symptom List Scores

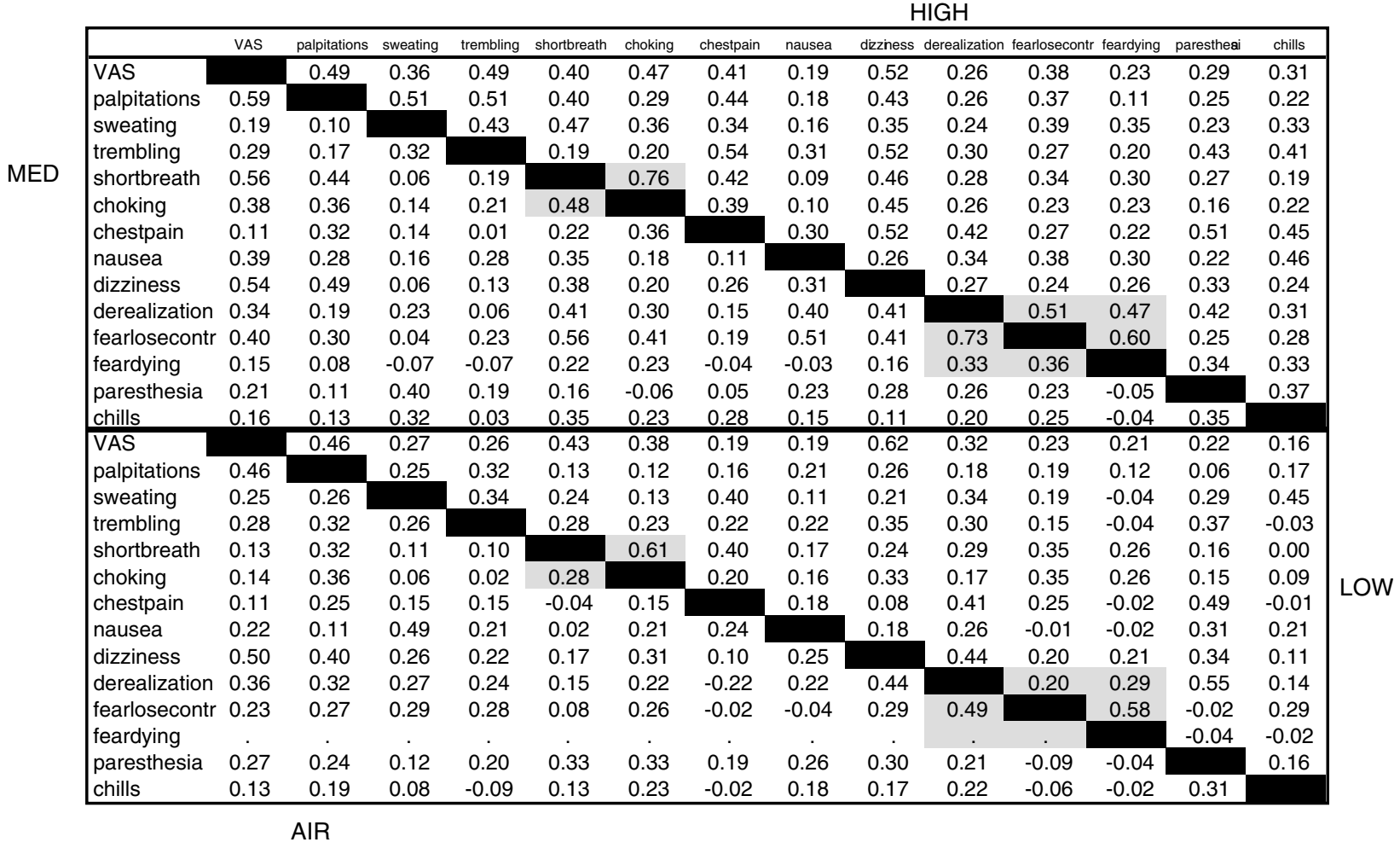

Spearman's correlation coefficients between DSM panic symptoms in four different $\mathrm{CO}_{2}$ conditions. 


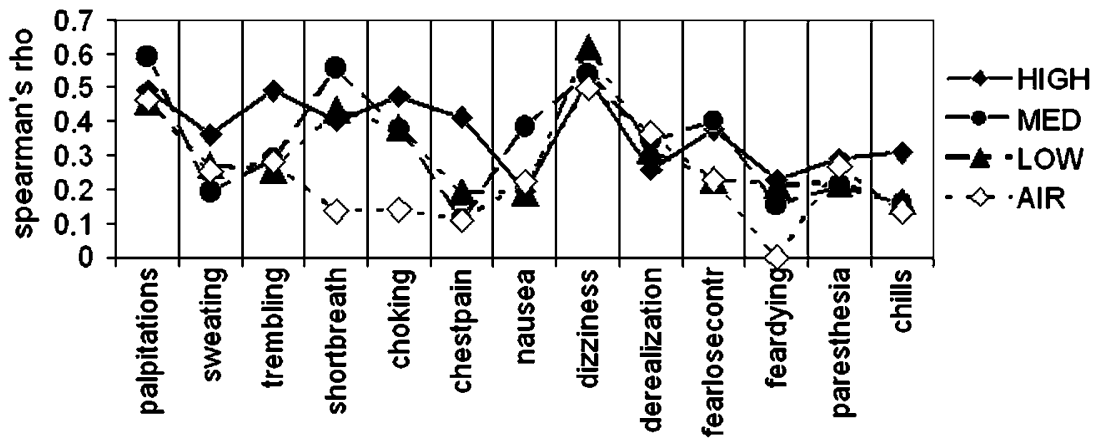

Figure I Correlations between Panic Symptom List scores and eVAAS Fear/Discomfort scores. Spearman's correlation coefficients between DSM panic symptoms and fear/discomfort scores in four different $\mathrm{CO}_{2}$ conditions. Sensation of shortness of breath and feeling of choking show a high correlation coefficient in all of the $\mathrm{CO}_{2}$ (high, med, low) conditions but not in the air condition.

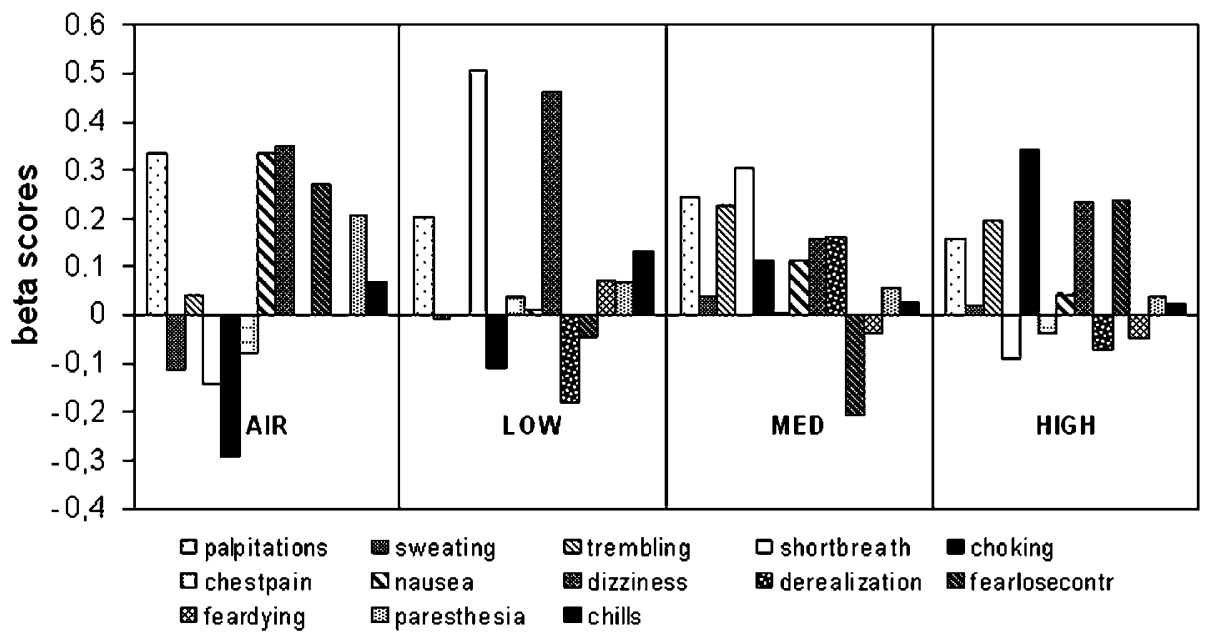

Figure 2 Regression analysis. Dependent variable: eVAAS fear/discomfort score. Relative weight ( $\beta$ scores) of separate DSM panic symptoms in predicting the intensity of fear/discomfort after the inhalation of 0\% (air), 9\% (low), 17.5\% (med), and 35\% (high) $\mathrm{CO}_{2}$.

Table 3 Factor Analysis of Panic Symptom List Scores

\begin{tabular}{lccc}
\hline & Respiratory & Cognitive & Neuro vegetative \\
\hline Shortness of breath & 0.856 & & \\
Feeling of choking & 0.845 & & 0.475 \\
Dizziness & 0.606 & & \\
Sweating & 0.582 & & 0.419 \\
Palpitations & 0.551 & & \\
Fear of dying & & 0.906 & \\
Fear of losing control & & 0.868 & \\
Derealization & & 0.744 & 0.781 \\
Trembling & & & 0.756 \\
Chest pain & 0.421 & & 0.697 \\
Chills & & & 0.649 \\
Nausea & & & 0.642 \\
Paresthesias & & & \\
\hline Prinipal component anlysi of DSM pani syptoms (ppomed & & \\
\hline
\end{tabular}

Principal component analysis of DSM panic symptoms (performed only in 35\% $\mathrm{CO}_{2}$ condition). Three clusters are extracted: respiratory, cognitive, and neurovegetative. Only factor scores $>0.4$ are shown.

included 'dizziness', 'sweating', and 'palpitations'. The cognitive component was defined by 'fear of dying', 'fear of losing control', and 'derealization'. The neurovegetative component was primarily characterized by 'trembling' but also included 'chest pain', 'chills', 'nausea', and 'paresthesias'.

\section{Regression Analysis (2)}

The three principal factor scores were then computed as variables. Regression analysis of factor scores as predicting variables (respiratory, cognitive, neurovegetative) showed that the respiratory component was a better predictor of eVAAS than the cognitive and the neurovegetative components $(\beta$ standardized coefficients $\beta=0.541 ; 0.391 ; 0.178$, respectively). The model explained $45 \%$ of the total variance $(R=0.67)$.

\section{DISCUSSION}

In the present study, we analyzed the phenomenology of the $\mathrm{CO}_{2}$-induced reaction in healthy volunteers. The symptoms reported by the subjects after the inhalations could be clustered into three distinct components. This confirms the first hypothesis that panic symptoms can be subgrouped in discrete clusters. The clusters were labeled 'respiratory', 'neurovegetative', and 'cognitive', according to the most prominent symptoms. The respiratory cluster was determined mainly by the feelings of shortness of breath and choking (second hypothesis). Moreover, a strong relation 
was demonstrated between the respiratory symptoms and the subjective feeling evoked by the $\mathrm{CO}_{2}$ challenge, which, according to the DSM criteria for PA, was labeled 'fear or discomfort'. Our third hypothesis was that respiratory symptoms were the best predictor of the subjective disturbance defined in the DSM IV criteria for panic. The $\mathrm{CO}_{2}$-induced emotion was specifically correlated with the respiratory sensations, and these symptoms were indeed the best predictors of the subjective fear/discomfort.

Fear and discomfort are two separate emotional entities but they appear together in the definition of panic attack provided by DSM-IV (APA, 2000). Consistently, to adhere as strictly as possible to the current psychiatric nosology, the subjects were asked to indicate the amount of the subjective experienced feeling, either it was fear or discomfort. The subjects were not instructed to specifically recognize the type of emotion they were feeling, to minimize a cognitive interpretation of their emotional experience.

The factor analysis of the present study extracted three components. The respiratory component was mainly characterized by shortness of breath and choking; it also included dizziness, sweating, and palpitations. The neurovegetative component was primarily characterized by trembling, but also included chest pain, chills, nausea, and paresthesias. The cognitive factor was characterized by fear of dying, fear of losing control, and derealization.

To the best of our knowledge, this is the first factor analysis performed on $\mathrm{CO}_{2}$-induced panic symptoms in healthy subjects. Previous reports on PD patients (Briggs et al (1993); Argyle and Roth (1989); De Beurs et al (1994); Cox et al (1994); Meuret et al (2006); Shioiri et al (1996)) generally agreed on several clusters, one representing a respiratory component. The similarity between our clusters, extracted in a normal sample, and clusters found in studies on PD patients supports the idea that the $\mathrm{CO}_{2}$ challenge in healthy volunteers may be a valid model to study the phenomenology of clinical panic.

Specifically, the most recent analysis by Meuret et al (2006) found a cardio-respiratory cluster that is strikingly similar to our respiratory cluster with regard to the presence of dyspnea, feeling of choking, and palpitations. However, it is slightly different for other symptoms, like dizziness and sweating, which, in Meuret's PD patient sample, were included in the autonomic/somatic cluster. Instead, in our study sweating, dizziness, and palpitations loaded on the respiratory component. Sweating showed high coefficients of correlations to many other panic symptoms (Table 2) and might represent an unspecific reaction, which occur in presence of general distress, including cases of respiratory distress. Palpitations may be considered related to a cardio-respiratory dimension, since changes in heart rate and cardiac output can be secondary to changes in respiratory drive. Dizziness may be considered part of a hyperventilation cluster therefore it may be explainable why it loaded on the RESPIRATORY component in our study. Moreover, dizziness might also be secondary to a transient cerebral hypoperfusion, secondary to bradycardia. Unfortunately we do not have any physiological measurement in the present study however, according to the studies of Argyropoulos et al (2002) and Kaye et al (2005) on the effects of $\mathrm{CO}_{2}$ in healthy volunteers, the expected cardiovascular reaction to $\mathrm{CO}_{2}$ consists in a bradycardic response accompanied by an activation of the sympathetic system with an increase in blood pressure. The bradycardia appears to be regulated independently of the pressor response and most likely involves the direct activation of brainstem vagal nuclei by $\mathrm{CO}_{2}$. Palpitations might also derive from the same changes in heart rate and the consequent activation of the baroreceptor reflex. Therefore, since both dizziness and palpitations are strongly related to the activation of the autonomic system, it may be explainable why a substantial secondary loading for dizziness and palpitations was also present on the neurovegetative component (Table 3).

The majority of factor analyses performed on clinical samples found that fear of dying loaded with the cardiorespiratory symptoms (Briggs et al (1993); Argyle and Roth (1989); De Beurs et al (1994); Cox et al (1994); Meuret et al (2006)), while in the present analysis it was the core symptom of the cognitive cluster. Such a difference may reflect a different cognitive elaboration of cardio-respiratory symptoms by PD patients compared to a non-clinical sample in that most PD patients tend to 'catastrophize' bodily symptoms whereas healthy subjects are not expected to have developed this cognitive pattern.

As far as the third hypothesis is concerned, the most important finding of the study was that of the respiratory component as the best predictor of the negative affective response to $\mathrm{CO}_{2}$ challenges. $\mathrm{CO}_{2}$ inhalation significantly induced almost every panic symptoms listed in the DSM IV. However, we showed that subjective fear/discomfort was specifically associated with an increase in respiratory symptoms. Feeling of choking, shortness of breath, dizziness, and palpitations had the highest scores (mean score $>1$ in the high condition) and the highest correlation coefficients with regard to the $\mathrm{CO}_{2}$-evoked subjective disturbance, represented by the eVAAS score. Yet, while dizziness and palpitations were related to the eVAAS score in every condition, including the air condition, the respiratory symptoms had high correlation coefficients specifically in the $\mathrm{CO}_{2}$-enriched conditions, that is, only in the low, medium, and high conditions.

Most of the clinical studies referred to above have provided support for the idea that respiratory symptoms are clinically relevant in $\mathrm{PD}$. The respiratory subtype of panic has a specific profile of pharmacological sensitivity, it is associated with familial risk of PD, and it may predict a greater severity of illness (Briggs et al (1993); Nardi et al, 2003, 2006a, b; Biber and Alkin, 1999; Onur et al, 2006). Epidemiological studies indicate that the presence of respiratory disorders, such as COPD (Goodwin and Pine, 2002), increases the likelihood of developing PD. Moreover, the link between panic and respiration is strengthened by the finding that PD patients have higher levels of irregularity and complexity of respiratory patterns (Caldirola et al, 2004).

Some authors have suggested that not only respiratory symptoms, but also the cognitive cluster, represented by catastrophic misinterpretations, seems to correlate quite well with the severity of PD (Telch et al, 1989; McNally et al, 1995; Cox et al, 1991; Vickers and McNally, 2005). However, as the authors themselves admit, a possible limitation of those studies is the retrospective self-report design, which may have overestimated cognitive symptoms. In addition, 
even if the severity of cognitive misinterpretations does correlate with the severity of the disorder, the direction of causality from cognitive misinterpretations to the severity of PA has not yet been convincingly demonstrated. It is perfectly conceivable that the development of catastrophic misinterpretation may be secondary to the severity of attacks, rather than the severity of attacks being secondary to the self-generated misinterpretations.

Having demonstrated, that in experimental panic, subjective fear/discomfort is more correlated to respiratory sensations than to cognitions, we provide support for the idea that respiratory control, rather than cognitions, plays a major role in the pathophysiology of panic. An interesting hypothesis put forward by Klein some years ago (Klein, 1993; Preter and Klein, 2008) inferred that PAs might derive from an abnormal 'suffocation alarm', an evolved, survivaloriented, inner physiological mechanism that erroneously signals the lack of useful air and the danger of impending suffocation, thus leading to urgency to breathe.

If, indeed, from an evolutionary point of view, panic emerged as an emotion to alert the subject of a biological danger, then it seems logical that a subjective sensation of panic (or discomfort) goes along with the severity of respiratory sensations. This is exactly what we observed in our results, which perfectly fit the core idea proposed by Klein, but also fit the function of emotions as expressed by such authors as Damasio (1999) and Panksepp (1998).

One apparent peculiarity of our results may need further comment. We observed that, in the high condition, 'feeling of choking' was the best predictor of the fear/discomfort score while 'sensation of shortness of breath' appeared to be a poor predictor (Figure 1). The opposite behavior was observed in the low condition. The importance of choking in predicting the eVAAS score increased with the dose while the importance of shortness of breath decreased. Recently, Ietsugu et al (2007) examined the relationship between the severity of PA and individual symptoms reported by a clinical sample. Shortness of breath was a predictor of moderate severity of PAs, while choking was a good predictor of high severity. Our results in experimental panic seem to be in line with Ietsugu's results in clinical panic. It may be proposed that the feeling of choking is more closely related to a stronger sense of suffocation, which is more likely to be evoked by the higher doses, while the sensation of shortness of breath may reflect milder symptoms of dyspnea.

In conclusion, the present study explored the phenomenology of an experimental model of panic in healthy volunteers. We found that the discrete clusters of symptoms observed in this study were similar to those elicited in naturally occurring panic. Consistent with the idea that respiration plays a crucial role in the pathophysiology of panic, we found that respiratory symptoms best predicted the subjective state defined in the DSM IV criteria for panic.

\section{ACKNOWLEDGEMENT}

We thank Dr Massimo C Mauri for the helpful suggestions and support. This study was carried out as a part of a research program in the context of the International Master of Affective Neuroscience.

\section{DISCLOSURE/CONFLICT OF INTEREST}

Dr Alessandro Colasanti discloses no conflicts of interest. No financial support or compensation has been received from any individual or corporate entity over the past 3 years for research or professional service and there are no personal financial holdings that could be perceived as constituting a potential conflict of interest.

Dr Ewa Salamon received a doctoral programme grant from the Warsaw Medical University for the academic year 2005/2006 and the research grant from the IESE Business School, University of Navarra, for the academic years of 2006/2007, 2007/2008. The related subventions were dedicated to the projects not associated to the submitted work. Besides the mentioned, no financial support or compensation has been received from any individual or corporate entity over the past 3 years for research or professional service and there are no personal financial holdings that could be perceived as constituting a potential conflict of interest.

Prof. Koen Schruers discloses no conflict of interests. No financial support or compensation has been received from any individual or corporate entity over the past 3 years for research or professional service and there are no personal financial holdings that could be perceived as constituting a potential conflict of interest.

Prof. Rob van Diest discloses no conflict of interests. No financial support or compensation has been received from any individual or corporate entity over the past 3 years for research or professional service and there are no personal financial holdings that could be perceived as constituting a potential conflict of interest.

Dr Marlies van Duinen received a grant in the Casimir programme 2007 from the Netherlands Organisation for Scientific Research (NWO) to accomplish a study not related to the current work. Besides the mentioned, no financial support or compensation has been received from any individual or corporate entity over the past 3 years for research or professional service and there are no personal financial holdings that could be perceived as constituting a potential conflict of interest.

Prof. Eric J Griez discloses no conflict of interests. No financial support or compensation has been received from any individual or corporate entity over the past 3 years for research or professional service and there are no personal financial holdings that could be perceived as constituting a potential conflict of interest.

\section{REFERENCES}

Abrams K, Rassovsky Y, Kushner MG (2006). Evidence for respiratory and nonrespiratory subtypes in panic disorder. Depress Anxiety 23: 474-481.

American Psychiatric Association (2000). DSM-IV-TR Diagnostic and Statistic Manual of Mental Disorders, 4th edn, Text Revision American Psychiatric Association: Washington, DC.

Argyle N, Roth M (1989). The definition of panic attacks, Part I. Psychiatr Dev 7: 175-186.

Argyropoulos SV, Bailey JE, Hood SD, Kendrick AH, Rich AS, Laszlo $\mathrm{G}$ et al (2002). Inhalation of $35 \% \mathrm{CO}(2)$ results in activation of the HPA axis in healthy volunteers. Psychoneuroendocrinology 27: 715-729. 
Aronson TA, Logue CM (1988). Phenomenology of panic attacks: a descriptive study of panic disorder patient's self-reports. J Clin Psychiatry 49: 8-13.

Bass C, Kartsounis L, Lelliott P (1987). Hyperventilation and its relationship to anxiety and panic. Integr Psychiatry 5: 274-291.

Battaglia M, Ogliari A (2005). Anxiety and panic: from human studies to animal research and back. Neurosci Biobehav Rev 29: 169-179.

Biber B, Alkin T (1999). Panic disorder subtypes: differential responses to CO2 challenge. Am J Psychiatry 156: 739-744.

Bouwer C, Stein DJ (1997). Association of panic disorder with a history of traumatic suffocation. Am J Psychiatry 154: 1566-1570.

Briggs AC, Stretch DD, Brandon S (1993). Subtyping of panic disorder by symptom profile. Br J Psychiatry 63: 201-209.

Caldirola D, Bellodi L, Caumo A, Migliarese G, Perna G (2004). Approximate entropy of respiratory patterns in panic disorder. Am J Psychiatry 161: 79-87.

Cox BJ, Endler NS, Swinson RP (1991). Clinical and nonclinical panic attacks: an empirical test of a panic-anxiety continuum. J Anxiety Disord 5: 21-34.

Cox BJ, Swinson RP, Endler NS, Norton GR (1994). The symptom structure of panic attacks. Compr Psychiatry 35: 349-353.

Damasio A (1999). The Feeling of What Happens: Body and Emotion in the Making of Consciousness. Harcourt Brace: New York.

de Beurs E, Garssen B, Bouikhuisen M, Lange A, van Balkom A, Van Dyck R (1994). Continuous monitoring of panic. Acta Psychiatr Scand 90: 38-45.

Goodwin RD, Pine DS (2002). Respiratory disease and panic attacks among adults in the United States. Chest 122: 645-650.

Gorman JM, Kent JM, Sullivan GM, Coplan JD (2000). Neuroanatomical hypothesis of panic disorder, revised. Am J Psychiatry 157: 493-505.

Griez E, Lousberg H, van den Hout MA, van der Molen MG (1987). $\mathrm{CO}_{2}$ vulnerability in panic disorder. Psychiatry Res 20: 87-95.

Griez EJ, Colasanti A, van Diest R, Salamon E, Schruers K (2007). Carbon dioxide inhalation induces dose-dependent and agerelated negative affectivity. PLoS ONE 2: e987.

Horwath E, Adams P, Wickramaratne P, Pine D, Weissman MM (1997). Panic disorder with smothering symptoms: evidence for increased risk in first-degree relatives. Depress Anxiety 6: 147-153.

Ietsugu T, Sukigara M, Furukawa TA (2007). Evaluation of diagnostic criteria for panic attack using item response theory: finding from the National Comorbidity Survey in USA. J Affect Disord 104: 197-201.

Kaye JM, Corrall RJ, Lightman SL (2005). A new test for autonomic cardiovascular and neuroendocrine responses in diabetes mellitus: evidence for early vagal dysfunction. Diabetologia 48: $180-186$.

Klein DF (1993). False suffocation alarms, spontaneous panics and related conditions. Arch Gen Psychiatry 49: 282-288.
Ley $R$ (1992). The many faces of Pan: psychological and physiological differences among three types of panic attacks. Behav Res Ther 30: 347-357.

McNally RJ, Hornig CD, Donnell CD (1995). Clinical versus nonclinical panic: a test of suffocation false alarm theory. Behav Res Ther 33: 127-131.

Meuret AE, White KS, Ritz T, Roth WT, Hofmann SG, Brown TA (2006). Panic attack symptom dimensions and their relationship to illness characteristics in panic disorder. J Psychiatr Res 40: 520-527.

Moynihan JE, Gevirtz RN (2001). Respiratory and cognitive subtypes of panic. Preliminary validation of Ley's model. Behav Modif 25: 555-583.

Nardi AE, Nascimento I, Valenca AM, Lopes FL, Mezzasalma MA, Zin WA et al (2003). Respiratory panic disorder subtype: acute and long-term response to nortriptyline, a noradrenergic tricyclic antidepressant. Psychiatry Res 120: 283-293.

Nardi AE, Valenca AM, Lopes FL, Nascimento I, Veras AB, Freire RC et al (2006a). Psychopathological profile of $35 \% \mathrm{CO}_{2}$ challenge test-induced panic attacks: a comparison with spontaneous panic attacks. Compr Psychiatry 47: 209-214.

Nardi AE, Valenca AM, Mezzasalma MA, Lopes FL, Nascimento I, Veras $\mathrm{AB}$ et al (2006b). 35\% Carbon dioxide and breath-holding challenge tests in panic disorder: a comparison with spontaneous panic attacks. Depress Anxiety 23: 236-244.

Onur E, Alkin T, Tural U (2006). Panic disorder subtypes: further clinical differences. Depress Anxiety 24: 479-486.

Panksepp J (1998). Affective Neuroscience, the Foundations of Human and Animal Emotions. Oxford University Press: London.

Preter M, Klein DF (2008). Panic, suffocation, false alarms, separation anxiety and endogenous opioids. Prog Neuropsychopharmacol Biol Psychiatry 32: 603-612.

Schruers K, Klaassen T, Pols H, Overbeek T, Deutz NE, Griez E (2000). Effects of tryptophan depletion on carbon dioxide provoked panic in panic disorder patients. Psychiatry Res 93: 179-187.

Shioiri T, Someya T, Murashita T, Takahashi S (1996). The symptom structure of panic disorder: a trial using factor and cluster analysis. Acta Psychiatr Scand 1996 93: 80-86.

Telch MJ, Lucas JA, Nelson P (1989). Nonclinical panic in college students: an investigation of prevalence and symptomatology. J Abnorm Psychol 98: 300-306.

Valenca AM, Nardi AE, Nascimento I, Zin WA, Versiani M (2002). Respiratory panic disorder subtype and sensitivity to the carbon dioxide challenge test. Braz J Med Biol Res 35: 783-788.

Van Duinen MA, Rickelt J, Griez EJ (2008). Validation of the eVAAS. Prog Neuropsychopharmacol Biol Psychiatry (in press).

Verburg K, Griez E, Meijer J, Pols H (1995). Respiratory disorders as a possible predisposing factor for panic disorder. $J$ Affect Disord 33: 129-134.

Vickers K, McNally RJ (2005). Respiratory symptoms and panic in the National Comorbidity Survey: a test of Klein's suffocation false alarm theory. Behav Res Ther 43: 1011-1018. 